\title{
INNER MAGNETOSPHERE VARIATIONS AFTER SOLAR PROTON EVENTS. OBSERVATIONS ON MIR SPACE STATION IN 1989-1994 TIME PERIOD
}

\author{
Ts.P. Dachev ${ }^{1}$, J.V. Semkova ${ }^{1}$, Yu.N. Matviichuk ${ }^{1}$, B.T. Tomov ${ }^{1}$, R.T. Koleva ${ }^{1}$, P.T. Baynov ${ }^{1}$, \\ V.M. Petrov', V.V. Shurshakov², Yu. Ivanov², \\ ${ }^{1}$ Solar-Terrestrial Influences Laboratory,e-mail: stilrad@bgcict.acad.bg \\ ${ }^{2}$ Institute of Biomedical Problems, Moscow, Russia, e-mail: shurshakov@mmcc.ibmp.rssi.ru
}

\begin{abstract}
Measurements on board the Mir space station have been used to study the dose rate and the particle flux distribution in the inner magnetosphere. The measurements have been performed with the BulgarianRussian dosimeter-radiometer Liulin. The paper concentrates on the dynamics of the observed "new" and "second" maxima which were created after Solar Proton Events (SPE) in the 1989-1994 time. The "second" belt was first observed after the SPE on October 20, 1989, and the last observation was after the SPE on February 20, 1994. The creation of the "new" belt is a unique phenomena seen in the Liulin data set after the SPE on March 23, 1991 and relates to the magnetic storm on March 24. The new belt fully disappear in the middle of 1993.
\end{abstract}

\section{EXPERIMENTAL RESULTS}

The Bulgarian-Russian dosimeter-radiometer Liulin Dachev et al (1989) was placed permanently in the working compartment of the big diameter inside Mir space station. The instrument was designed to measure the dose and flux of penetrating particles simultaneously and uses a silicon detector with a thickness of 306 microns and an area of $2 \mathrm{~cm}^{2}$. The effective mass thickness of shielding matter for working compartments of Mir space station is evaluated to be between 6 and $15 \mathrm{~g} / \mathrm{cm}^{2}$. Thus the main contribution to the count rate are given by protons and electrons that have energies outside Mir space station more than $100 \mathrm{MeV}$ and $10 \mathrm{MeV}$ respectively.

\section{$\underline{\text { SPE on } 29 \text { September } 1989}$}

The instrument did not operate during the first hours of the SPE. First data were recorded by Liulin at about UT=19:00 in the southern hemisphere. The proton flux at $\mathrm{L}>3.5$ increased up to 150 particles $/ \mathrm{cm}^{2} \mathrm{~s}^{1}$ and the dose reached values of about $2 \mathrm{mGy} / \mathrm{h}$, which is the highest dose record by Liulin in the time interval 1989-1994. Some of the phenomena observed during this SPE are published in Dachev et al (1992) and by Lobakov et al (1992). The instrument was operated until October 10, 1989, but remarkable new creations in the inner magnetosphere were not observed. 
Liulin recorded in real time the onset and the initial phase of this flare. The measurements includes observations of multi-GeV solar particles at the station altitude in low and equatorial latitudes (Smart et al.,1994). In Figure 1 a brief overview of the data is presented. The three graphics give the dose in $(\mu \mathrm{Gy} / \mathrm{h})$, flux in (particles $/ \mathrm{cm}^{2} \mathrm{~s}$ ) and the dose to flux ratio in (nGy $\mathrm{cm}^{2} /$ particle). On the $\mathrm{x}$ - axes 176 sequential data points are presented and the time resolution between two different points is 100 seconds. Each maximum in the dose and flux curve corresponds to one pass of the station through the high latitude regions of both hemispheres and each minimum to the station pass at low and equatorial latitudes. It is seen that in the UT interval 13:26 - 15:01 at low and middle latitudes the flux increases to about $10-12$ (particles $/ \mathrm{cm}^{2} \mathrm{~s}$ ). At the same time and place there is no drastical dose increase. The analysis of the dose to flux ratio shows that the energy (dose) deposition per particle at low latitudes in this time period is very low and may be explained by the presence of protons with energies of a few $\mathrm{GeV}$ or by albedo electrons.

Due to a lack of data an additional maximum was first observed in the inner after the SPE on 25.10.1989. In Figure 2 the data are presented on dose, flux and dose to flux ratio obtained on 27.10.1989. between 16:57 and 17:42 UT. On the $X$ axes 270 sequential data points are presented and the time resolution between two different points is 10 seconds. The bottom panel presents the $\mathrm{L}$ value of the station. The station orbit crosses the region of SAA south-east from the place of the main maximum (M) which is located in the center of Figure 2 at $\mathrm{L}=1.4$. The second maximum seen may be explained by an additional particle injection to the inner magnetospere following the SPE resulting in an additional radiation belt. From the dose to flux ratio we conclude that protons are predominantly registered in this maximum. The second maximum at $\mathrm{L}=3$ was observed permanently until the end of operation of Liulin in 1989 (10.11.1989). The position of the second maximum suggests that it is comparable to the second radiation belt which was first reported by McIlwain (1963).

In the operational period of Liulin between 05.09.1990 and 22.03.1991 no additional maximum was observed at $\mathrm{L}$ values below 3 .

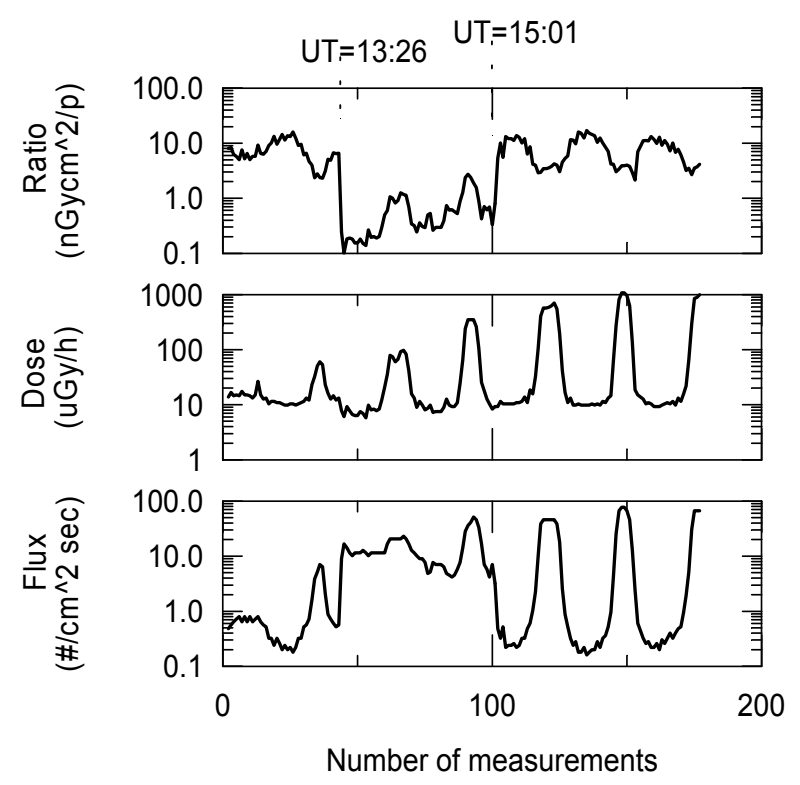

Fig. 1. Initial phase SPE data on 19.10.1989. The dose to flux ratio shows that the specific dose deposition at low latitudes in the 13:26 15:01 time period may be explained by the presence of protons with energies of a few $\mathrm{GeV}$ or by albedo electrons.

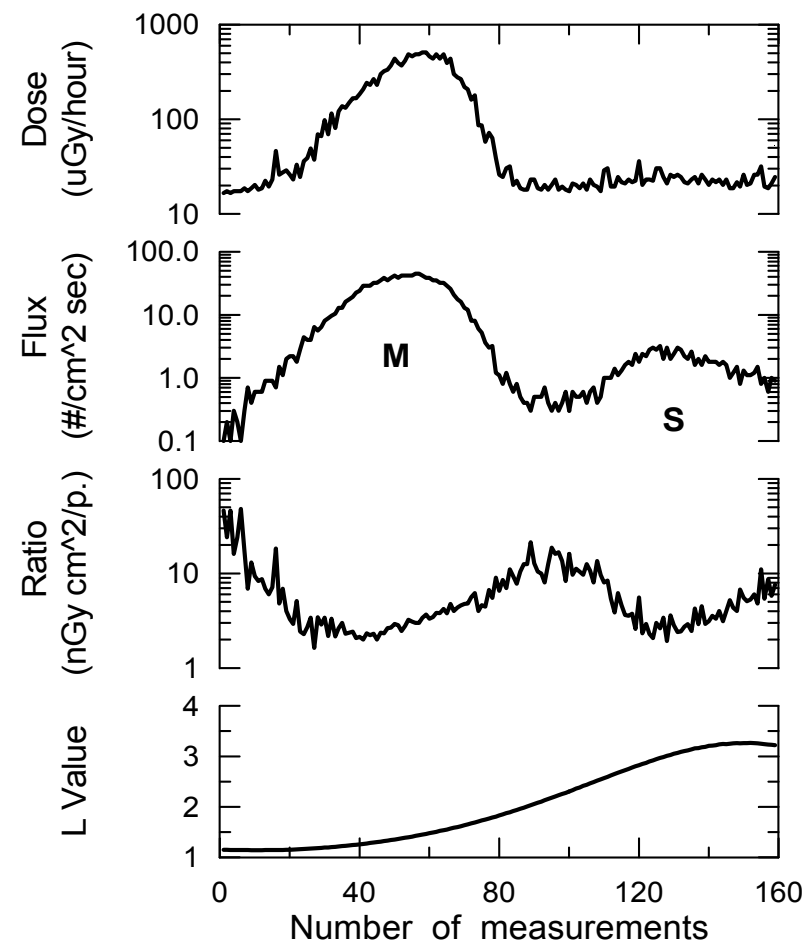

Fig. 2. Observations of second radiation belt maximum at $\mathrm{L}=3$. It may be explained by an additional particle injection to the inner magnetospere following the SPE. 


\section{SPE on 22 March 1991}

First measurements started at 12:07 UT. Since the station was located at low $\mathrm{L}$ values no remarkable phenoma could be observed. The flux of solar protons on 23.03.1991 at 20:50 UT at L>3.5 was considerably lower than on 29.09.1989 and does not exceed 8 particles $/ \mathrm{cm}^{2} \mathrm{~s}$. Results concerning this event are published by Petrov et al (1993), Petrov et al (1994) and Dachev et al (1995).

Measurements at low latitudes after the SPE and the following magnetic storm show that drastic changes have occurred in the inner magnetosphere. Two additional maxima appeared. The maximum at $\mathrm{L}=3$ seems to be the same phenomena as seen in October 1989, and can be assigned to the second radiation belt while the maximum $(\mathrm{N})$ at $\mathrm{L}=2.2$ is completely new in the Liulin data. Predominant particles in these two maxima evaluate from protons to electrons. The maximum at $\mathrm{L}=3$ slowly disappeared until the beginning of May 1991. This features are presented in Figure 3. where data are presented for dose, flux and dose to flux ratio obtained on 01.04.1991 between 00:24 and 01:11 UT ploted on the $\mathrm{X}$ axes by 270 sequential data points with time resolution $10 \mathrm{sec}$ between two different points. It is well seen that the main maximum in both dose and flux curves has additional "steps" on the west and east sides, which can be interpreted like a superposition of the three maxima. From the dose to flux ratio we can conclude that the "steps" are generated by protons which have a lower energy than those in the main maximum, while the new maxima are generated predominantly by electrons.

\section{$\underline{\text { SPEs in June } 1991}$}

The particle flux and the dose rate in all three maxima was increased after the solar activities between 1-15 June 1991. The measurements along one orbit on June 14 and 20, 1991, are shown on Figure 4 and Figure 5, respectively. In Figure 4 the data are plotted for periods of time on 14.06.91. 18:17-19:02 UT. In Figure 5 the data are plotted for time period of 20.06.91 between 15:49 and 16:34 UT. The path of both orbits are practically the same which corresponds to similar L coordinates seen at the bottom of the figures. The width of the additional maximum in flux is more than $2000 \mathrm{~km}$ (crossed by the station in 4 minutes) that is many times greater than the gyro radius of trapped protons with an energy of $100 \mathrm{MeV}$ at $400 \mathrm{~km}$ altitude. The increase

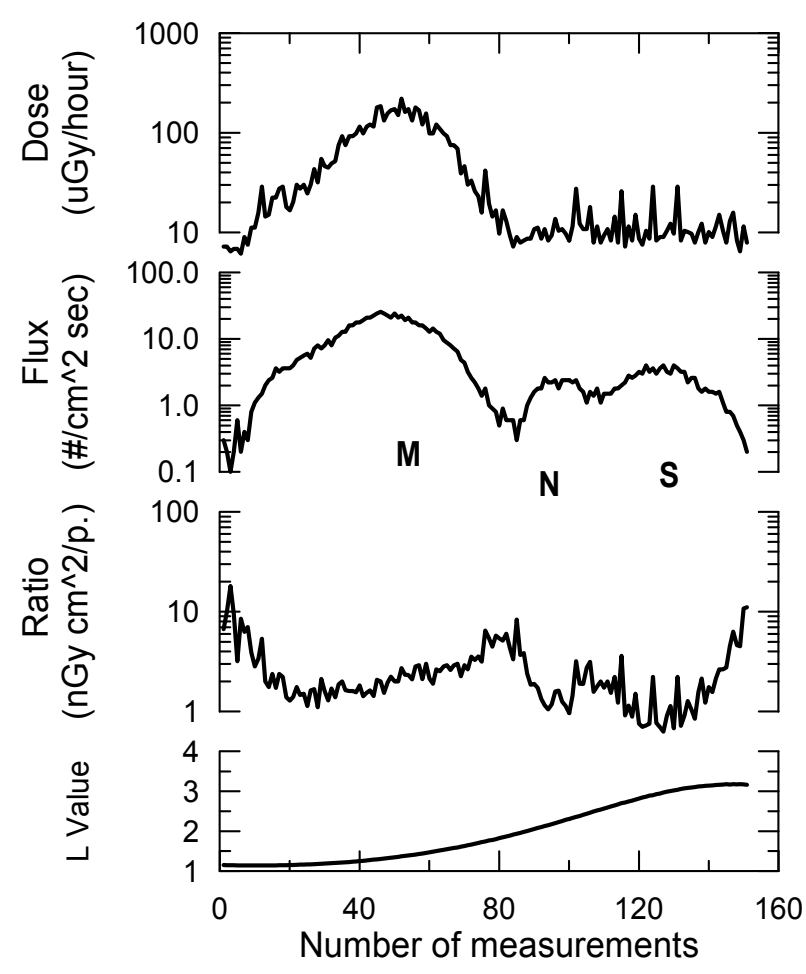

Fig. 3. Variations after SPE on 23.03.1991. Three different maxima are seen: Main (M), New (N) and Second (S)

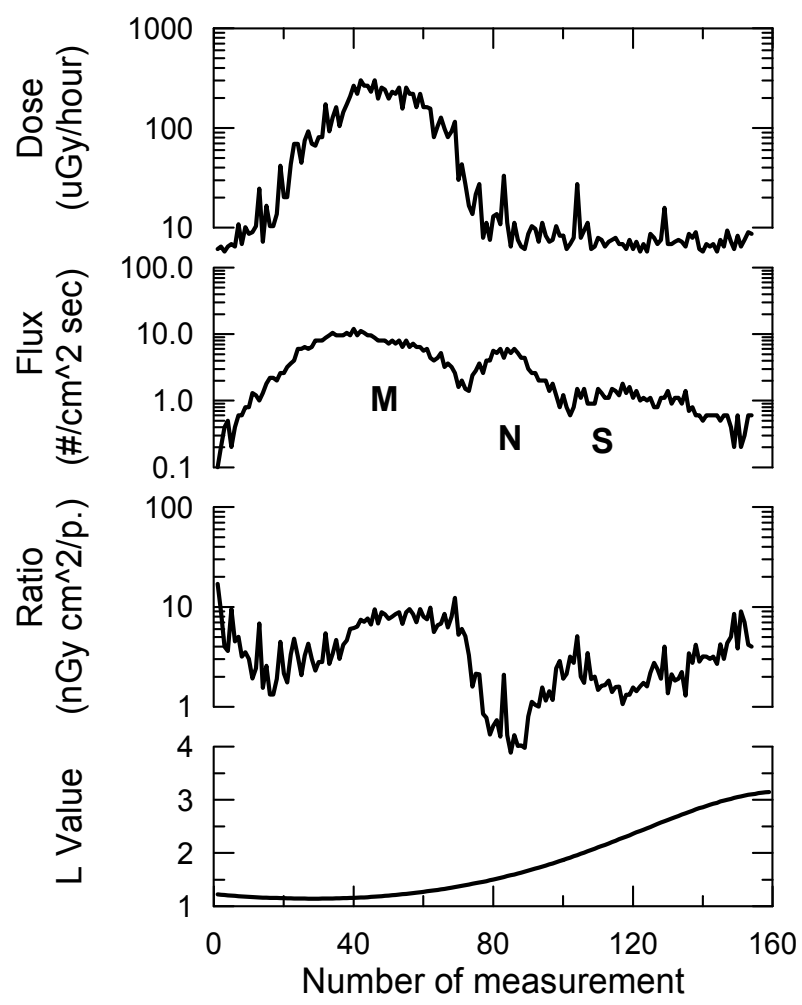

Fig. 4. Liulin data for 14.06.1991 which shows relative low levels of all observed maxima 
of the doses and fluxes seen in Figure 5 is probably caused by an additional "pumping" of diffusing particles from solar origin during the SPE on 15 and 16 June into the inner magnetosphere. One important result of the SPE on 15 June is the increase of the main proton maximum by factor of 2 . The sequence of this increase is presented in Figure 6. The data were taken inside the main SAA maximum in the period 14-29 June 1991. There is an increase in dose and flux in the main maximum from 14 to 26 June 1991 followed by a decrease until 29.06.1991. In the same time a continuous decrease of the dose to flux ratio was observed. This can be explained by the increase of the mean energy of the protons which are creating the dose in the instrument. The $\mathrm{L}$ value of all parts of orbits is about the same.

Figure 7 shows the variation of the new maximum. There is the same behaviour in flux as for the main maxima, but the dose is at the maximum at the left hand site of the peak and drops already down before the flux reached the maximum. This can be understand when at the left hand site of the maximum the protons dominate and at the right hand site the electrons. This can be well understood by using Figure 8 where the dose to flux ratio in the main and second maxima are presented in dependence of flux. It is seen that the amounts of points for protons and electrons are well separated and the separation increases with the increase of flux. The dotted lines are calculations of the dose to flux ratio of protons of different energies and electrons by Haffner (1971).

\section{$\underline{\text { SPE on } 22 \text { February } 1994}$}

After this $\mathrm{SPE}$ a second maximum at $\mathrm{L}=3$ was again observed. The predominant particles in the second maximum were electrons. The second maximum was observed continuously until the end of available Liulin data on Mir (beginning of May 1994.)

\section{CONCLUSIONS}

The main conclusions for the dynamics of the inner magnetosphere observed by Liulin behind more than 6 $\mathrm{g} / \mathrm{cm}^{2}$ of shielding in 1989-1994 time period after SPE activities are as follows:

Up to three maxima were observed, the main, the new and the second maxima. The main maximum is situated

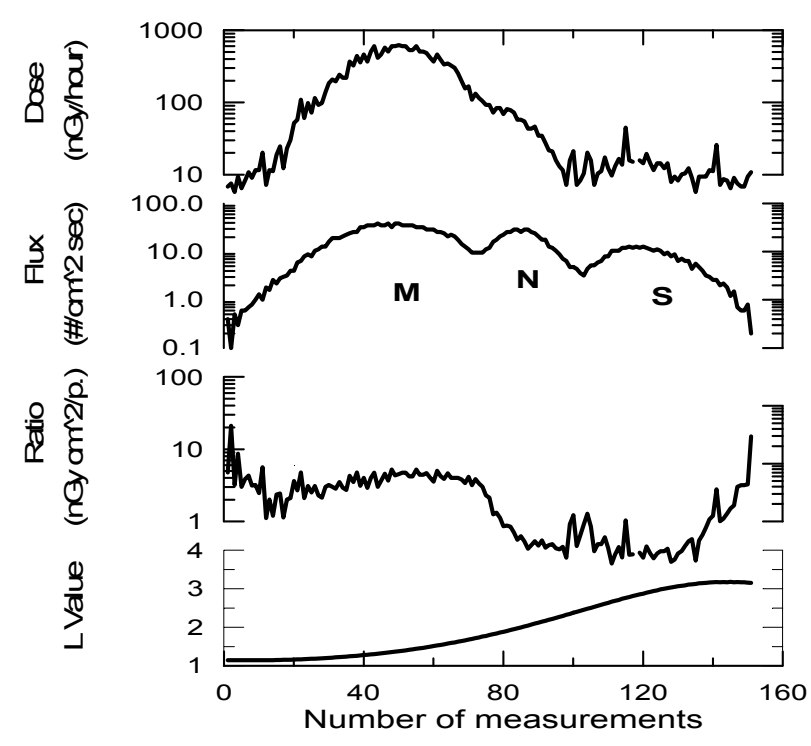

Fig. 5. Liulin data for 20.06.1991. All three maxima are increased in comparison with data presented on Fig. 4.

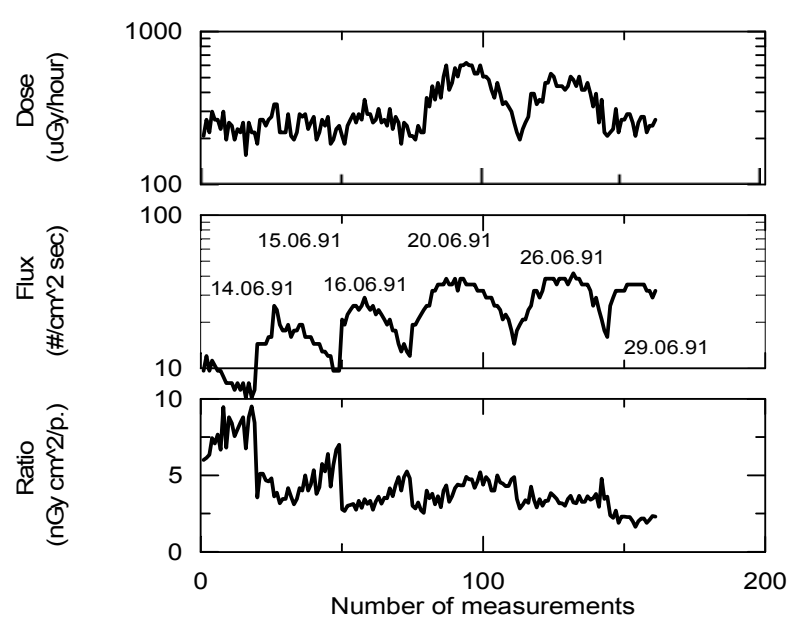

Fig. 6. Main maxima variations in June 1991

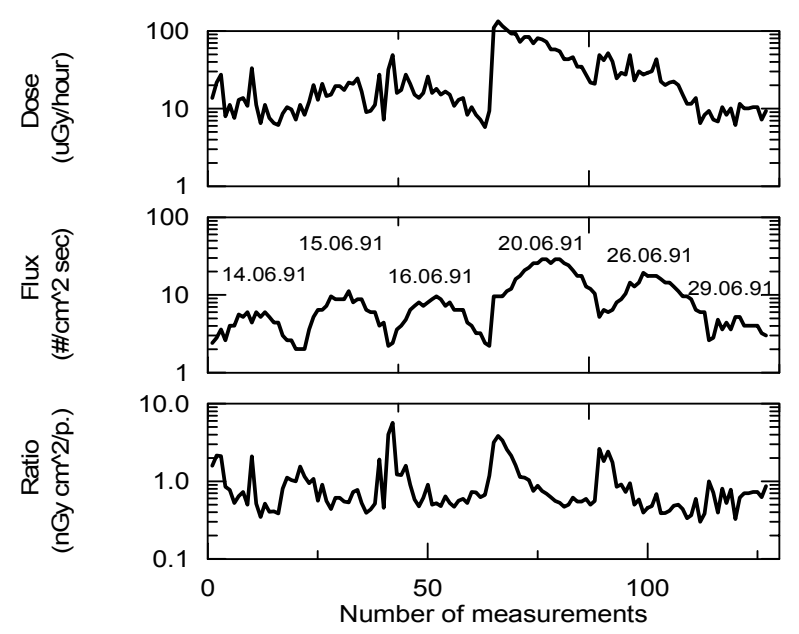

Fig. 7. New maximum variations in June 1991 
in the SAA at $\mathrm{L}=1.3-1.5$. This maximum is observed always and consists mainly by protons. The dose rate varies by an order of magnitude from 200 to 2000 $\mu \mathrm{Gy} /$ hour dependent of the shielding, altitude and time after SPE. The flux varies in the interval of 30-120 particles $/ \mathrm{cm}^{2}$ s. Major changes of the main maximum together with the appaerence of two additional maxima occured after the SPEs of 22 March 1991, 14 June 1991 and 20 June 1991. After the SPE on 14 June 1991 the main maximum rose up by afactor of two and decayed again until 30 June 1991.

The second maximum (in other classification outer radiation belt) is not so stable like the main and is located at

$\mathrm{L}=3$. It was intensified after some of the SPSs during the period (19 October 1989, 22 March 1991, 15 June 1991 and 20 February 1994). The dose rate in it varied by a factor of 2 from 8 to $16 \mu \mathrm{Gy} / \mathrm{h}$. The flux varies between 0.8 and 12 particles $/ \mathrm{cm}^{2} \mathrm{~s}$. The dominant particles in the second maxima are protons close after the SPE and electrons later. The second maximum decayed in a few months. For example after the SPE on 22 March 1991, it disappeared completely until 15 May. Additional studies are necessary to answer the question, why some SPE intensified it and others do not.

The new maximum was created after the SPE on 22March 1991, and was located between the main and the second maxima at $\mathrm{L}=1.8-2.2$. After the solar-terrestrial disturbances in June 1991, and especially after the SPE on 15 June 1991, it was remarkable intensified. When it was first created it was completely separated by the main, and the predominant particles were electrons. Later in June it moves toward the main maximum and the predominant particles in it are protons on the west bound side (side to the main) and electrons on the east bound side, that is why the dose rate in it varied by factor of 12.5 from 8 to 200 $\mu \mathrm{Gy} /$ hour. The flux varied between 0.8 and $30 \mathrm{part} / \mathrm{cm}^{2} \mathrm{~s}$. The new maximum decayed toward the main from June 1991 until middle of 1993.

The intensity of the inner magnetosphere variations is not a linear function of the intensity of the solar protons during the main phase of SPE at L values more than 3.5. 


\section{REFERENCES}

1. Dachev, Ts. P., Yu. N. Matviichuk, J. V. Semkova, R. T. Koleva, B. Boichev, P. Baynov, N. A. Kanchev, P. Lakov, Ya. J. Ivanov, P. T. Tomov, V. M. Petrov, V. I. Redko, V. I. Kojarinov, R. Tykva, Space radiation dosimetry with active detections for the scientific program of the second bulgarian cosmonaut on board the Mir space station, Adv. Space Res., 9, 10, 247, 1989.

Dachev, Ts. P., Yu. N. Matviichuk, N. G. Bankov, Ya. J. Ivanov, B. T. Tomov, V. M. Petrov, V. I. Redko, M. V. Zil, V. G. Mitrakas, T. N. Smirnova, V. V. Temny, Yu. N. Ponomarev, R. Tykva, "Mir" Radiation Dosimetry Results during the Solar Flares events in September-October 1989, Adv. Space Res., 12, 2-2, (2)321-4, (1992).

Dachev, Ts.P., J.V.Semkova, Yu.N.Matviichuk, R.T.Koleva, B.T.Tomov, P.T.Baynov, J.F.BottollierDepois, V.D.Nguen, L.Lebaron-Jacobs, M.Siegrist, E.Duvivier, B.Almarcha, V.M.Petrov, V.V.Shurshakov, New results for the space radiation environment of MIR space station obtained by LIULIN dosimeter-radiometer. Comparison with LET spectrometer NAUSICAA, Acta Astronautica, 36 505-515, (1995).

Haffner, J.W., Nuclear radiation and protection in space, 114, Moscow, Atomizdat, (1971). (in Russian)

Lobakov,A.P., V.I.Liagushin, M.P.Panasiuk, P.I.Shavrin, V.S.Kchomutov, V.M.Petrov, V.A. Shurshakov, T.P.Dachev, J.V.Semkova, Increase of solar cosmic rays on the "MIR" Space station in orbit during September-October 1989, Nuclear Tracks and Radiation Measurements, 20, 1, 59, (1992).

McIllwain, C.E., The radiation belts natural and artifical, Science, 142,355, (1963).

Petrov V.M., Machmutov V.S., Shurshakov V.A., Panova N.A., Dachev Tz. Space distribution of particle flux and absorbed dose rate in the South Atlantic Anomaly Region based on measurements made on board of Mir space station, Izvestiya Rossiyskoy Akademii Nauk, Seriya fizicheskaya, 57, No. 7, PP. 100103, (1993).

Petrov,M.V., V.S.Machmutov, N.A.Panova, V.A.Shurshakov, Ts.P.Dachev, Ju.N. Matviichuk, J.V.Semkova, Pecularities of the Solar Proton Events of October 19, 1989 and March 23, 1991 According to the Measurements On Board the MIR Space Station, Adv. Space Res., V 14, No.10, (10)645, (1994).

Smart,D.F., M.A.Shea, Ts.P.Dachev, N.G.Bankov, V.M.Petrov, V.V.Bengin, The Dose Rate Observed on 19-21 October 1989 and its Modulation by Geophysical Effects, Adv. Space Res., V 14, No.10, (10)651, 1994. 